\title{
Exploring the Relationship between Buddhist Mindfulness Meditation, Wellbeing and Personal Goal
}

\author{
Ven. Sajjana, Kumar, Gautam Anand, Arvind Kumar Singh
}

\begin{abstract}
The empirical application of Buddhist mindfulness meditation suggests by this notion of impact had stood through considerable development over two thousand and five hundred years ago. In this field, the exploration propounds the outcomes of mindfulness practice along with using survey method as well as the evolution of Buddhist mindfulness practice to around the world as treating psychological development. The target of the survey will suggest to the practitioners who are currently joining at the mindfulness meditation classes in the various meditation center of India and Myanmar. In this field, to collection of the most practical benefits of mindfulness will be examined in detail whatever we have got the result from survey. To deal with above mentioned issue, this field had tried to clarify the results from respondents who are practicing mindfulness in their experience as well as we have been trying to practice with them for some moments. The present paper will emphasize the discussing about Buddhist mindfulness and the impact of practicing mindfulness meditation.
\end{abstract}

Keywords : Mental Suffering, Anxiety, Stress, Physical Diseases, Empirical Benefits of Mindfulness, Sati or Satipatthana (Foundation of Mindfulness), Chart or Figure.

\section{INTRODUCTION}

Nowadays, in the globalization, the materialism seems to havebeen influencing people around the world with newer material thing every day, while the world of idealism or the spiritual things have been leaving behind increasingly. As people are joying along with the development, simultaneously, human beings have been facing along with many problems from many corners of the world. The cause of problemsthat may be found from the outside world is that the most countries have tried to develop in their material issues. So many countries try to fulfill their people with all material which they could provide, that could lead to various kinds of conflict in all around the world, while leaving the spiritual world behind. From since more than 25 centuries,mindfulness meditation discovered by Buddha has been playing as the important role in helping beings of the universe in moderating their spiritual world so that they can develop, moderate, share and help each other able to livealong with harmony anddeveloped minds.In this field, we

Revised Manuscript Received on July 22, 2019.

Ven. Sajjana, Research Scholar, Sharda University, Noida (Uttar Pradesh), India.

Dr. Kumar, Gautam Anand, Assistant Professor, Sharda University, Noida (Uttar Pradesh), India.

Dr. Arvind Kumar Singh, Assistant Professor, Gautam Buddha University, Gautam Buddha University, Noida (Uttar Pradesh), India. would like to introduce about mindfulness meditation. Mindfulness meditation is most helpful for human lives. One can take mindfulness meditation for solution various problems. Especially, it seems to be understanding of lives and to be getting peaceful spiritual life. The mindfulness mediation can absolutely help everyone who wants to be joyful peace in their daily life. The regular spiritual practice of mindfulness meditation can help to awareness of spiritual values in human's daily lives.

Especially, people who always use their energy for their living; whether seeing, hearing, or thinking, whenever one uses the six sense organs the entire body's fiery energy will naturally become concentrated in the head, burning up the entire body's watery energy.So, Meditation is a practice that aims to remove all these deluded mind and relevance in daily life through to bring down all the fiery energy, and to up the pure watery energy. As this survey stands, the field has made the topic"Effect of Mindfulness Meditation in daily life."It means if one takes mindfulness meditation, how to influence good things his or her life by this taking meditation, what is benefit of meditation in their daily life and how many effects have they got by meditating? This survey research about mindfulness meditation is quite limitedthat is only for people who are practicing mindfulness meditation for sometimes from ten days to up, not for all.

\section{OBJECTIVE OF THIS RESEARCH SURVEY}

The aim of this paper especially survey is to explore the relationship between mindfulness meditation, well beings and personal goal and to evaluate the significance of mindfulness for all has been marking the peaceful life in social environment by mindfulness meditation. It is also to studyabout evaluation of mental and physical health development and gradually evolution of mindfulness treatment in psychological aspect into the western world. To be discovering the practical benefits, in this field, willbe examined the relevance of mindfulness meditation in human's daily life.

\section{Methodology}

In thisfield, we used classification of data from earlier respective sources able to verify regarding this research paper and collection of data through questionnaires method and interview method. Especially, we used the collection of data through schedules method which 
lies in the fact that schedules are being filled by the enumerators who are specially appointed for the purpose.

Schedules go to respondents and put to them the questions form to answer and the questions are listed and record the record the replies in the space meant for the same answers.

\section{Evolution of Western Psychological Treatment USING BUdDHIST MINDFULNESS}

In the western culture, the psychological treatment had been proliferated since the last two centuries, the eighteenth and nineteenth centuries. The stemming acceptance within mainstream western culture as the method or promoting mental health is often rooted in beliefs that western psychotherapy is universal in its healing ability due to its use of science (Sidhu, 2017). In 1733, the very first book of psychotherapy called 'The English Malady, or A treatise on nervous diseases of all kinds. As spellen, vapours, lowness of spirits, and hysterical distempers, etc.' was published in London, written by George Cheyne who was a famous physician from Scotland. The aim for the mental patients, Anton Mesmer from France discovered the treatment of magnetism. Academy of Sciences of France did not accept this theory after investigating to treat mental patients. In the nineteenth century, Sigmond Freud from Austria, Charocot from Frane, and James Braid from England used to this magnetism as the other named called hypnotism to treat mental patients (Nissanka, 2018).

In the western world, England had led to the establishing mental hospitals showing their humane manner. From the royal college of surgeons, James Birch Sharpe led to as a chairperson in a select committee to recommend measures to improve the treatment of mental patients in 1815. Other English men who are Emil Kraeplin and EugenBleuler, Tuke and Bucknill published a text book called 'Psychological Medicine'. Among them, Kraepelin investigated his exploration of mental patients as three forms which are the first ones is dementia praecox (an illness which leads to disintegration of personality. This illness is now known as schizophrenia), the second form is manic depressive psychosis (a mental disorder which alternates between period of excitement and depression), and the third form is paranoia (an illness with delusions and persecutions), in 1882, (Nissanka, 2018).

During from 1885 to 1920 , the students of Freud, who are Carl Jung and Alfred Adler led to develop the psychoanalytic method along with a new approaching to psychotherapy. In the modern times, the establishment of American psycho-analytic association stands also a land mark of the development of psychotherapy (Nissanka, 2018). The whole century of nineteenth, in the western culture, the treatment of spiritual power had popularized for mental patients. The cause of becoming popular this treatment was that the evil spirits created the mental illnesses as a prevailing belief in the west tradition. Therefore, the spiritual power men who thought that they have power to drive away the spirits possessed were the only persons to whom the people could go. The common treatment was that they drill the skull of the patient and leave a hole so that the evil spirit could go out or escape. The physicians believed that the evil spirits were in the blood of the mental illnesses' person and they let out the patient's blood and infused blood from a health person. In the last two centuries, the mentioned above methods are taught in the faculties of medicine in the universities of east and west (Nissanka, 2018).

In the twentieth century, the healthy development of the west is that the observations have gone on two directions, the first one is scientific study on the mental disorders to determine the psycho physical conditions of specific mental illness and the causes that brought out those illness and the second is scientific study of the chemical reactions of psychotherapeutic drugs and their efficacy in restoring mental health of the patients. The humane in contrast to the practices means psycho-therapeutical treatment can be obtaining by people who are mental patients in the private and public clinics or hospitals. The several systems of psycho- analysis also had developed along with this treatment in the western culture. For post graduate students of medicine, Prof. Storr published the book named the art of psychotherapy. In this book, Storr mentioned that the place should begin in an appropriate consultation room and he divided the mental patients into the four groups such as the hysterical personality, the depressive personality, the obsessional personality and the schizoid personality (Nissanka, 2018).

In around 1980 and 1990, the Buddhist mindfulness meditation as a therapeutic practice in the west occurred and is attributed to the work of Jon KatbatZinn who is a professor emeritus of medicine and the creator of the stress reduction clinic and the center for mindfulness in medicines, health care, and society at the university of Massachusetts Medical School (Gethin, 2011). The systematical techniques of mindfulness therapy include Linehan's dialectical behavior therapy (DBT) system in 1993 and acceptance and commitment therapy (ACT) system of Hayes et al (1999). Among them ACT system less closely associate with specific psychological problems, but also the other therapies have been used more widely in the western tradition (Krageloh, 2013). Mindfulness practice is used by KabatZinn led to the development of his two therapies which are mindfulness-based stress reduction (MBSR) and mindfulness-based cognitive therapy (MBCT). Buddhist mindfulness was more about ridding oneself of greed and other negative qualities and in the west mindfulness is utilized to resolve stress and other specific mental health issues. The aim of KabatZinn build on the mindfulness practice emphasized to reduce stress as a practice that could be passed on in psychotherapy (Gazzola, 2016). Zinn declared how mindfulness is the most suitable method for dissolving this problem. By paying attention to the present moment, the sense of control is retained. The state of mindful provides an opportunity for self-reflection, self-growth, and self-awareness that previously was unavailable. The ability to process deep emotion consciously is liberating and empowering (Zinn, 1994). 
The mental patients can be understanding through therapy has incorporated by KabatZinn the concept of mindfulness meditation into a therapeutic practice and his therapeutical treatment (MBIs) mindfulness-based interventions have also been used as an important therapy for both mental and physical health issue. Mindfulness-based interventions (MBIs) aim to eradicate stress, anxiety, depression, eating disorders in the mental health. The method of Mindfulness-based stress reduction (MBSR) which is essentially mindfulness meditation practice along with the intent to relieve unwanted symptoms aims to use as treatment for both physical and psychological pain (Williams, 2015).

Prof. Daniel Siegel who is the clinical psychiatrist investigates the link between the brain and mind using mindfulness practice (Gazzola, 2016). Dr. Siegel found the notion that mindfulness practice is a sacred method for psychotherapy and he made the testing and explanation its function from a neurological standpoint. To discern between the activity of the mind and the individual's totality, Dr. Siegel explores the concept of mindfulness as the ability. He found the notion from mindfulness practice that the mindfulness individual identifies that these phenomena are merely passing by increasing mindful of sensations, images, feelings and thoughts (Siegel, 2007). According to his explanation, the mere realization that one cannot be liberating in himself but one investigates the brain for understand how these functions of mindfulness actually operate. Dr. Siegel encourages the future researchers to hypothesize how it can be best applied to physical and psychological well-being on a neurological level (Siegel, 2007). Focusing attention in specific ways activates the brain's function and regarding this way, mindfulness practice therapy essentially increases neuroplasticity (Siegel, 2007). For nervous system, one must be understand the central point that the mind is the highest place where thoughts and feelings occur and the brain is physical organ. Easily understanding is that the mind is software and the brain is hardware. In well-being of the mind, mindfulness practice creates the brain's changing that the mind changes the brain and the brain then affects how the mind functions. Mindfulness practice is the certain areas of the brain to activate. In the brain occurring, the physiological changing of function increases (Gazzola, 2016).

The goal of mindfulness-based cognitive therapy (MBCT) is more precisely on changing the way participants respond to their cognition. According to Dr. Siegel, through mindfulness practice, the patients learn to process cognition differently, preventing recurrence of depression when presented with new challenging situations. The patients start to observe the literal content of thoughts non-judgmentally and realize impermanent nature of those thoughts as well as the fact their content is not necessarily an accurate representation of reality. The structure of MBCT and MBSR is a very similarly program but MBCT is more effective than MBSR. In MBCT program, exercise method that are doing practical method to people with depression, such as observation of the association between mood and worried thoughts, is designed by Dr. Siegel (Evans et al, 2008).
KabatZinn (2003) asserts that the effectiveness of MBSR is not simply due to relaxation. Baer (2003) supports Zinn's declaration that patients with chronic pain are not simply taught techniques that directly target pain reduction, but instead learn to observe their sensations and emotions more closely as they happen in the moment. The patients are inspired to develop non-judgmental attitude to their sensation by simply observing them and continually returning their attention to present moment. These techniques are based on Theravada tradition call satipatthana meditation (mindfulness practice) emphasis on the beginning stages of foundation of mindfulness such as contemplation of body and feelings (Gilpin, 2008).

\section{Reduction of Mental Sufferings, Stress ANd ANXIETY}

Even though human's life consists of physical and mental, it can be understood that mind is forerunner and body is a follower. All human's problems and sufferings arise into the mind and the cessation of these suffering and problems lie in mind itself. The influence of mind as understood in the Buddhist way is such that mind is part of a psychological science which includes investigation into the mental activities of human life. Nowadays, in the western's society, psychology is applied to education, industry, business, health care, national defense as army, law, politics, sociology, science, art, and even sports increasing with time. The Buddha extended to have knowledge and realization the human who are searching for happiness avoiding suffering even though they really do not know what to do. Greeson. et al, (2011) used to try a survey for an eight week mindfulness based stress reduction (MBSR) program along with using the two emotion regulation strategies, decreased suppression and increased reappraisal. They found the interaction along with increased mindfulness of thoughts and feelings, and decreased symptoms of anxiety and depression.

Mindfulness meditation those are techniques like MBSR and other mindfulness based interventions has been shown to reduce negative mood induction and to alleviate negative emotional reactions from ruminative thoughts (Feldma et al. 2010). Decreased emotional reactivity to personally salient social stress induced in the laboratory has also been observed in MBCT (Britton et al. 2012). Negative thinking in the mode of rumination, worry, attachment and self-judgment seems a tool that produces negative affect. Negative thinking that is rumination can aggravate negative moods that feelings of sadness can induce into depression and momentary feelings of fear can feed longer lasting anxiety (Watkins, 2008). By normally observing thoughts, emotions, and other perceptions along with a sense of non-judgments and acceptance, one is able to develop increasingly comfortable exposing oneself to potentially upsetting thoughts, feelings, and even physical pain without trying to any change or control one's experience. Mindfulness practice has ability able to help individuals change how they relate to thoughts and feelings through several cognitive, emotional, 
psychophysiological, and behavioral processes.

Mindfulness meditation is aware of present moment but the present moment is not always a time of rest. Meditation can be helpful able to reduce the stress and soften anxiety. The benefits of the greater mindfulness practice develop as a result of unique steps which are the first step is that when one allows oneself to feel the worries, irritations, painful memories, and bad thoughts and emotions, mindfulness can be help to remove and reduce the feelings of difficulties. The second step is that mindfulness meditation allows able to safely explore the underlying causes of the stress and anxiety. By going to through this way, the explorer has a chance the opportunity to realize insight into what is driving human's concerns rather than expending energy fighting or turning away from anxiety. The third step is that when practitioner begins to understand the underlying causes of the apprehension, mindfulness meditation can help to create space around the apprehension as it does not absorb or as freedom and a sense of spaciousness naturally emerge. Bob Stahl (2010) mentions his attitude on mindfulness practice that 'practice mindfulness is a process of learning to trust and stay with feelings of discomfort rather than trying to escape from or analyze them'. This attitude is a part of the concept of Buddhist teachings. According to the Buddha teachings, the meditator must firstly observe the suffering to understand because of that without understanding of the object no one can escape from suffering (Majjimanikaya, Satipatthanasutta, 1954).

Mindfulness meditation is not overly created the nature and it can be found the basic human ability to be fully present moment which aware of where we are and what we are happing. When one becomes aware of the present moment, one may not be able to change your situation through mindfulness, but one can change his or her response to their situation (Kabat, Zinn, 1990). Segal et al., (1992) discovered the most popular practice which called the three minute breathing space into the eight week mindfulness based cognitive therapy program. This time has able to shift the practitioners' attention away from various thoughts do not stuck. This practice simply becomes mindful of various thoughts and feelings. Human often faced over the difficult emotions and mind states. Some scholars tried to mention reflect and underlying calculation that even though trying to avoid or push away negative thoughts and feelings can be exhausting.

MBCT program introduced regarding above mentioned systems as the some ways which one of those is permission to exist negative emotion in human's lives is called the concept of acceptance which means intention to experience for possibility of developing a different relationship. It means to understanding present situation before responding to them how to do. Another way is that practices of MBCT program indicate solid ways for cultivating an attitude of allowing and letting be among painful experiences. Even though we do not realize how to do it, these abilities are unlikely to be produced merely by an effort of will. Contemplating attention or awareness has possibility of learning to relate differently to achieve experiences in each moment that this practice of working through the body can allow people to realize and they can allow unpleasant experiences and still be okay (Segal, 1992).

All mental distress comes from one's own experience, thoughts and worries about future and the mind spinning out of control, making it difficult or impossible to concentrate and focus in the present. Anxiety also has emotional and physical aspects illuminated by feeling of fear. For reducing anxiety and stress, Bob Stahl (2010) encourages to practice mindfulness whatever position which it can be comfortable and alert such as standing, seating and lying down. Mindfulness can be a powerful method of working with anxiety and it amalgamates well along with approaches, including medications, psychotherapy, and counseling. Bob Stahl (2010) mentions some method to practice mindfulness these are that taking a moment to acknowledge oneself for being here means taking present time and go inside into one's own lives. Making connection with mind and body means to feel any sensations and any holdings in the body as well as feel into mood, feel into any emotions and just thank to being felt and letting be. Gently withdrawing the awareness from breathe means when one arises any sensations in the body, one will shift his or her focus to a body scan. Be allowing to any anxious thoughts that arise along with mindful inquiry means while contemplation mindfulness practice on the body and into the mind, there may arise any feeling such as anxious thoughts, worries, and fears. In this situation, one should continuously contemplate mindfulness to experience with inquiry able to discover potentially the underlying causes of the anxious thoughts. Taking a moment to watch your thoughts means practitioner is watching the breath in and out, simultaneously, one can watch any thoughts arising into the mind. When one is watching the thoughts as observing even the thoughts of fear, one can realize the realities all phenomena are coming and going, changing one by one. When one finds himself that one becomes aware of thoughts, one can become free from those anxious thoughts. Finally, one must be mindful breathe coming and going. Above mentioned ways, human can be reduced any anxiety and can be faced any challenged feelings.

\section{ANALYSIS OF SURVEY RESUltS}

This survey research had started through questionnaire method and schedules method. The starting time of survey, we have been jointedto centers of vipassna meditation centerin several places such as Mumbai, New Delhi and Dharamshala in India and all vipassana centers in Myanmar and met people who are practicing in various sections for some period. We have spent in place of meditation along with the practitioners for some time at the least about ten day sections. Than we were discussing about the questionnaires and collected back their answers and experiences. We have recordedinformation with different types of answers form respondents. For 
instance, in the mindfulness meditation classes, some male practitioners said that they practicedmindfulness meditation in everyday or for sometimes, some female said that they did it for less than 4 weeks in a month.

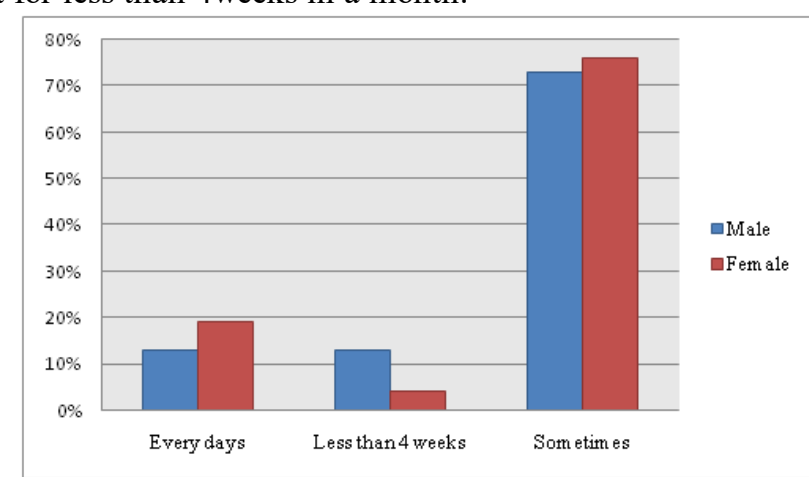

Fg.1 - Meditation times for normal practitioners in various places

Thechart Fg.1 shows that how long the peoplehave been practicing mindfulness meditation in a year in vipassana centers in accordance with each category. In number of 160 respondents, the $16 \%$ respondents are meditating mindfulness practicein everyday, $10 \%$ are in less than 4 weeks and $71 \%$ respondents are trying mindfulness meditation sometimes. Among them $61 \%$ are male and $39 \%$ are female. We have achievedthe result in different situation between male and female. 13\% of 97 respondents of male are practicing mindfulnessin everyday, another $13 \%$ also practicein less than 4 weeks in a year and $73 \%$ interest it sometimes. For female $19 \%$ of 63 respondents is taking mindfulness meditation in everyday and only $4 \%$ takes in less than 4 weeksin a year and $76 \%$ take it sometimes. In this case, the illustration had come across clearly that female practitioners are more interesting meditation than male. Of course, it should be happen because of mindfulness meditating can be moderating pressure of family and press of money and press of any suffering for those. The most of people make solution their pressure as practicingmindfulness meditation.

According to Buddhist tradition, there are many types of meditation but only contemplation breathe method (anapana meditation) has should be most suitable for this survey. Anapana meditation means observation of the natural breath coming in and going out. The most easy and comfortable way is not only for beginner but also for all practitioners. When one starts to practice meditation, he or she must be started from this breathing method and then one will go to higher stages step by step. As the taking meditation place, most of people are use in meditation centerbut a few percentage uses in their room. If we mention with numbers, $89 \%$ of 160 number respondents take meditation in meditation center and $11 \%$ of 160 respondents take it in their won room. Actually, this is not necessary for meditate, wherever and whenever one can practice meditation. Important is that one should practice meditation for peaceful and spiritual values.

As the religious, most mindfulness practitioners are non-Buddhist in India, mindfulness meditation is not only for Buddhist but also for all. A person who is what religious

is not important in this research survey. The purpose of mindfulness meditation will be demonstrated as result of why they take meditation and what is benefit they got from it? It mentions below chart Fg. 2 .

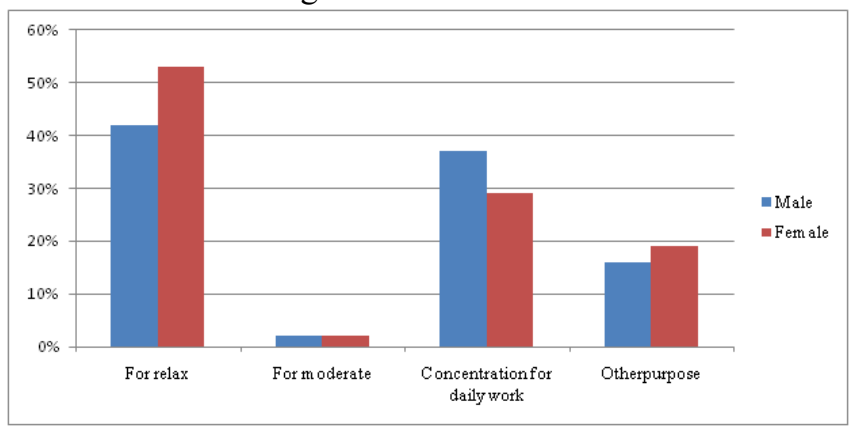

Fg.2 - Purpose of meditation based on gender

The Fg.2 mentions the purpose of meditation of respondents as show enumeration, $47 \%$ of 160 respondents practice mindfulness for relaxation, $2 \%$ of respondents for moderate, $34 \%$ is for concentration for daily work and $17 \%$ are for other purpose. When this survey divides in detail as gender, $42 \%$ of 97 male respondents mark meditation for relaxation, and $2 \%$ are for moderate, $37 \%$ take it for concentration for daily work and $16 \%$ of 97 male respondents take for other purpose that depending on them. As the female respondents, $54 \%$ of 63 respondents are for relax, $2 \%$ are for moderate, $29 \%$ are for concentration for their work and 19\% are for other purpose.

This field discovers the records of both male and female are practicing mindfulness meditation in order to concentrate for their happy lives. That should be right because all are human and they have found many stress along with jobs or works in their daily life. They could not finishquite

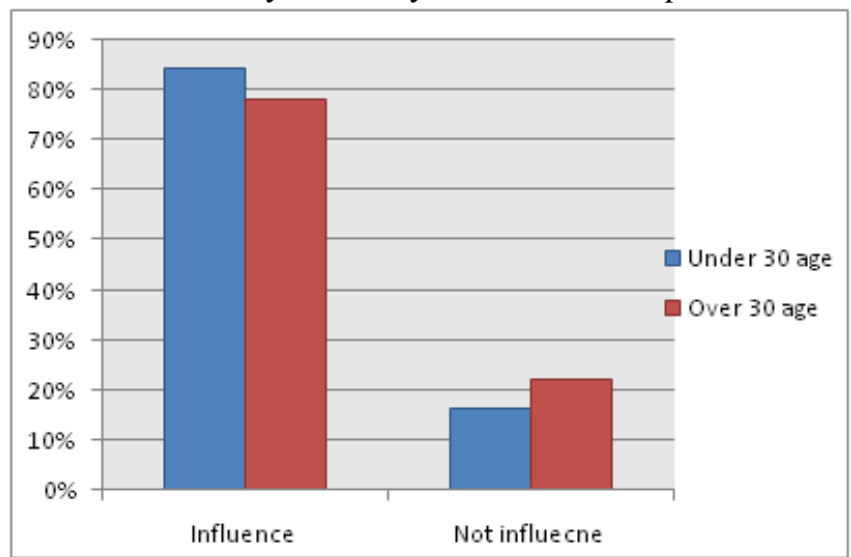

Fg.3 - Influence of daily life based on occupationswell with those pressures, thus, they need to remove or reduce these situations as practicing mindfulness meditation able to out of stress and get concentration and relax.Similarly, Buddhist or non-Buddhist and Indian or foreigner practice mindfulness for relax and concentration in their daily lives. It is not only getting relaxation and concentration for livelihood but also the influence of good situation in daily life. The results can be found in chart Fg.3.

According to Fg.3, all practitioners of respondents are saying 'yes' as agree with the influence of meditation in their daily life. $82 \%$ of 160

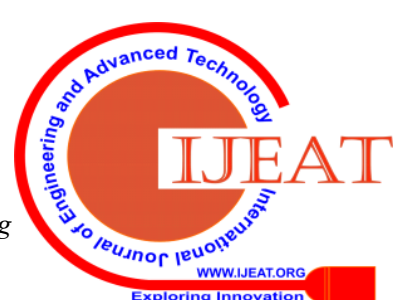


respondents are agreed with this subject. As in detail, $84 \%$ of 115 under thirty year old respondents and $78 \%$ of 45 over thirty year old respondents admit the good influence of mindfulness meditation in daily life.

This good influence is most benefit of meditation for them, and it can improve on their spiritual values. $16 \%$ of 115 under 30 ages and $22 \%$ of over 30 ages respondents are not accepted this case as they said 'no'. If this survey divides in gender, the result can be found in accordance with Chart Fg. 4 . The $76 \%$ of 97 male respondents and $79 \%$ of 63 female respondents are accepted the good result which mindfulness meditation can influence in their daily life. The $24 \%$ of male and $8 \%$ of female respondents are did not agree with in such as significance (Chart Fg.4).

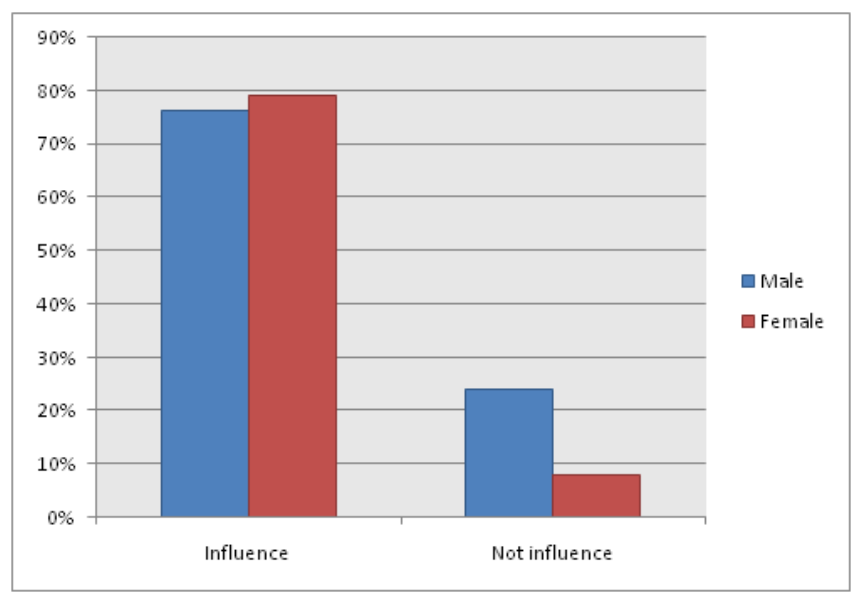

Fg.4 - Influence of daily life based on gender

When one executes his or her duty or something jobs, how does one determine in mind? Have they tried them as a function or with any purpose in mind? The answer arises that is depends on what is accepted situation on those works. Similarly, when one decides to practice mindfulness meditation into the whole life, the practitioner can accept as obligation or as a duty and other purpose such as free willing. In recording of this field with questionnaire survey method, several different purposes are found by people who are practicing mindfulness in daily life as the result of findingsomeone's purpose standsfor obligation and someone for it in order to free willing.

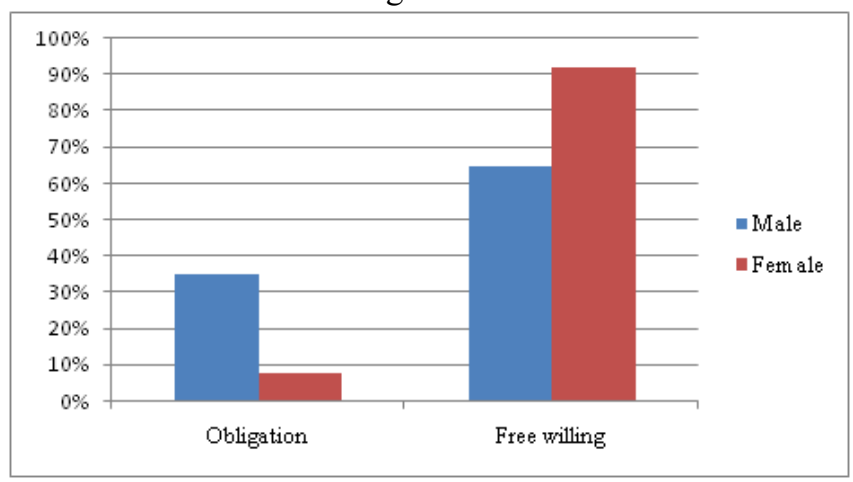

Fg.5 - Point of meditation based on gender

In the chart Fg.5, we found most of respondents are joining meditation for free willing. Numerous of percentages of them are hope to get free willing for achieving the highest well-being of mindfulness meditation. In this survey, we have investigated about the case into gender among various types of practitioners. The $65 \%$ of 97 male and $92 \%$ of 63 female respondents have been wished able to achieve free willing from mindfulness practice. The $35 \%$ of male and only $8 \%$ of female are practicing mindfulness meditation for obligation in accordance with someone's forcing to do even without they do not mind. Whatever situation or purpose of practicing mindfulness meditation, that is suitable for in their life as well as in their daily works.

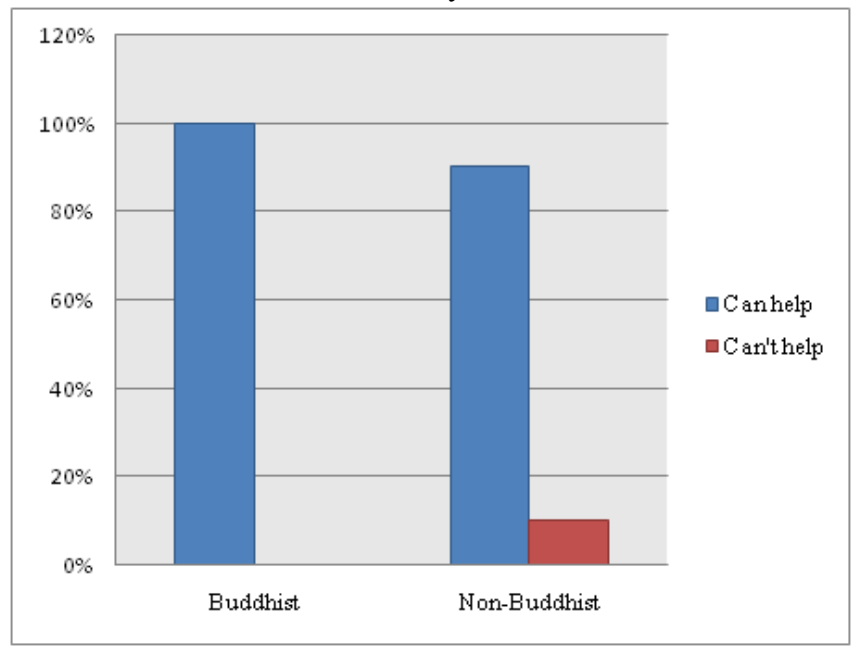

Fg.6 - The benefits of mindfulness meditation to daily life

This chart (Fg.6) indicates perfect result as to whether practicing mindfulness meditation could help living comfortably in daily life. As the chart showing result, several respondents, who are non-Buddhists, have mostly in their opinion that practicing mindfulness meditation could help them to live comfortably in their daily life along with more than $90 \%$ (percentages). There are only few of them denied, as $10 \%$, that they have no benefits for practice mindfulness. Whereas from the point of view of Buddhist, they all accepted that practicing mindfulness meditation could help them to live comfortably in their daily lives as $100 \%$.

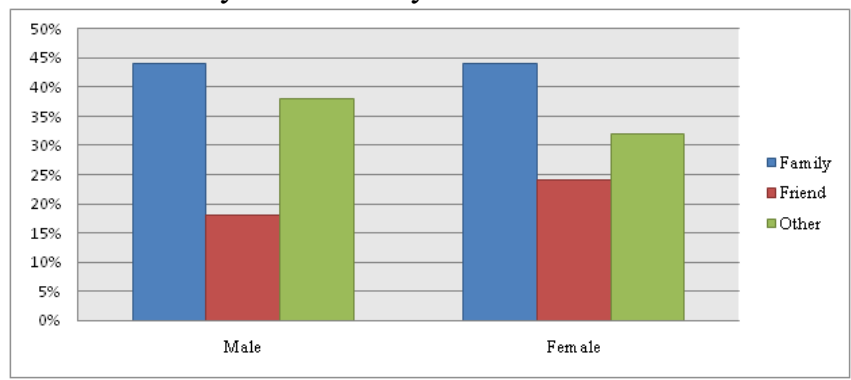

Fg.7 - Who play the most important role in knowing meditation?

As taking into account of Fg.7, the results discovered that the most influence one in knowing about meditation both male and female is "family" with $44 \%$ (percentages), as the other and friend can influence in lesser with $38 \%$ and $24 \%$ (percentages) respectively. In the promotion of taking meditation for practitioners (or anybody) families play the most important role. If families support or catch the sight of benefits of meditation and then introduce meditation to their children, it would be the powerful promoting way of 
meditation. In the Fg.7 indicates, it indicates the vital understanding in the human life that family plays the important rolethem not only in knowing meditation, but also in learning things that would be new for them.

When one would like to promote meditation, the family should be advised first. In other relevance dimension such as religion, home land, course and nationality, according to this survey, the families still play the most important role for them as well.

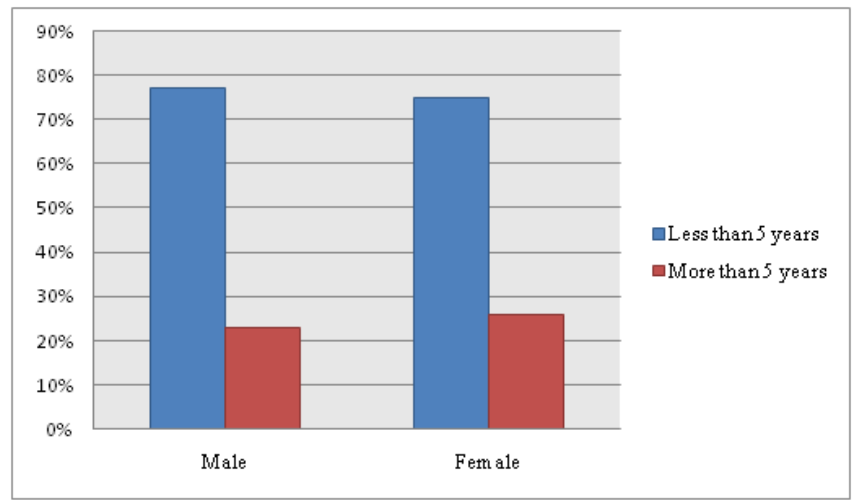

Fg.8 - Period of having been taking meditation based on gender

The chart Fg.8 mentions the remarkable information that both male and femalewho have been asking survey questionnaires in their daily lives by us as target group knew and have been practicing meditation less than five years. In the chart Fg.8, 77\% of male and $75 \%$ of female just having interest and practicing meditation less than five years. This information may looks strange for the other who could expect that they could mostly know about practicing meditation more than five years not less than 5 years as the result in this survey.There might be some things influence these practitioners as to why they have been taking meditation in such way.

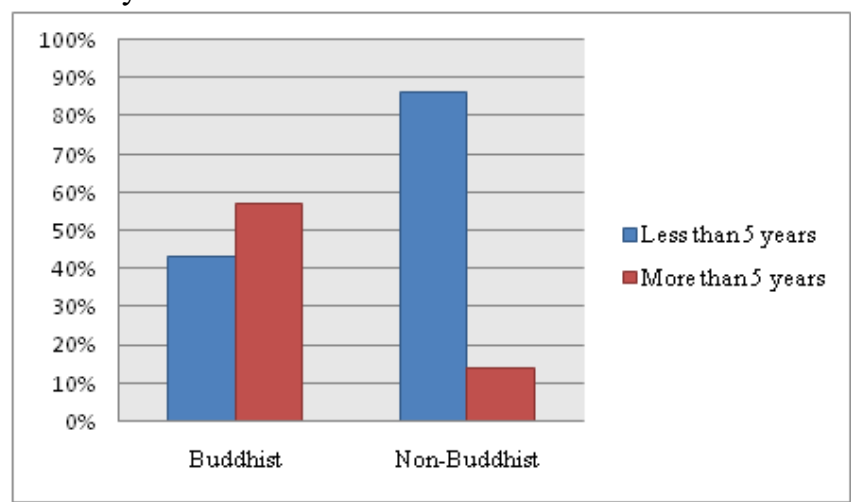

Fg.9 - Period of have been taking mindfulness meditation religious aspect.

As we have seen in chart Fg.8 before, practitioners in our target group have been joining meditation less than five years. So now it comes to another dimension that is of their background or cultures. The result has taking into account of the relevance of religion aspect which they will base on Buddhist and non-Buddhist aspect. According to the information of the period that practitioners, of course, in target of this survey, have been taking meditation, Buddhists have been joining meditation in their younger lives than
non-Buddhists that the chart Fg.9, indicates that non-Buddhist practitioners have been joining meditation less than five years with $86 \%$ as the Buddhist students have been joining meditation for less than five years only $43 \%$. It is double (the amount) as comparing with non-Buddhist meditators. While Buddhist meditators have $57 \%$ of having been practicing meditation, non-Buddhistshave got only $14 \%$ in their percentages of having been joining meditation more than five years. As above mentioned to have seen in previous (in Fg.8) that it could have something that we should contain into account of this matter as to what is the matter of fact that why non-Buddhist practitioners, which in this case, are most Indian and westerners come the range of taking meditation after their secondary life, which Buddhists seem to come into this area of joining earlier.

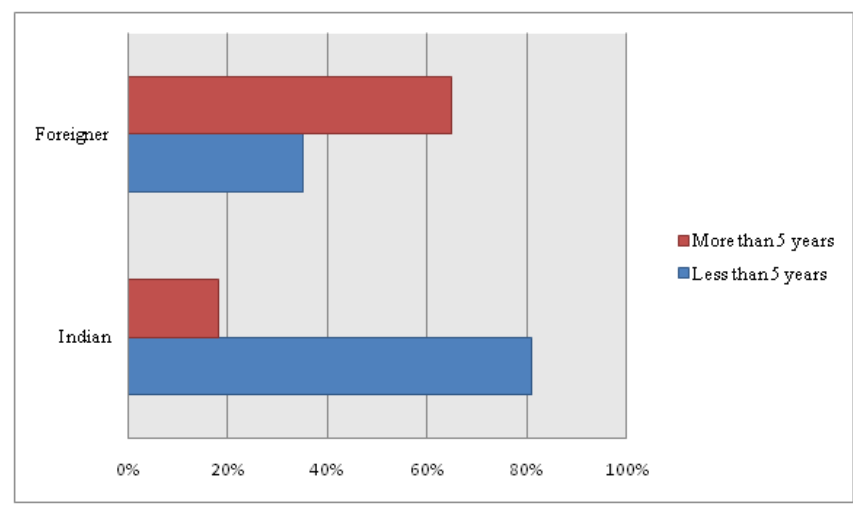

Fg.10 - Period of have been taking meditation on nationality aspect.

Wherefrom we have seen before, the first time of taking mindfulness mediation of practitioners in our target seem to come not in the same time as we take into account of their background such as gender, religion. Moreover, the survey is consideration to the matter that relates to practitioners' nationality.According to information from chart Fg.10, it indicates that Indian people seem to come in the range after foreigner with $81 \%$ of their joining less than five years, whereas foreigners, in this case, all of them are Buddhist and non-Buddhist, come in the range earlier with $35 \%$ for their joining. In the same way, we take consideration of having been practicing in part of mindfulness practitioners more than five years. Practitioners from foreign country have the more percentages of taking into practicing mindfulness before Indians along with $65 \%$ and $18 \%$ respectively. From this point it can be found that differences of the culture and tradition may have the relevance of this matter. To come into the part of practicing mindfulness meditation after or earlier is not the matter of taking meditation but the point and the benefits the results have been gotten from mindfulness meditation are the most important thing we should consider as the matter of fact. 


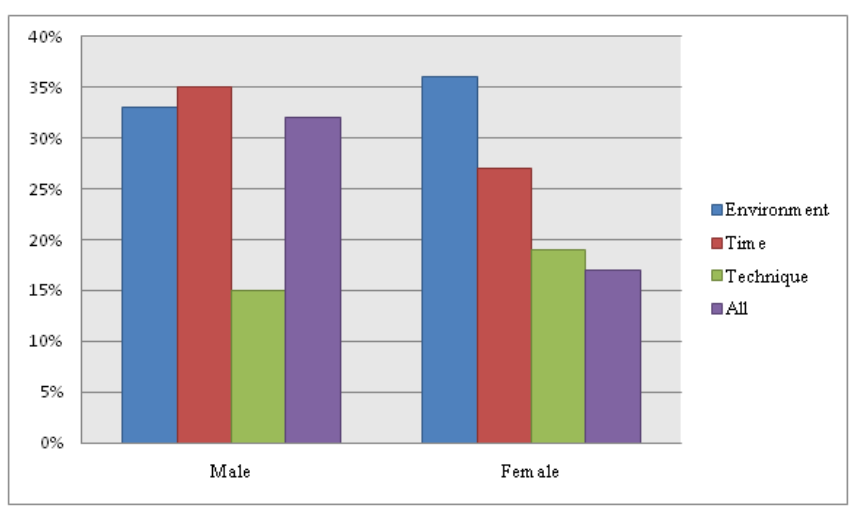

Fg.11 - Uneasiness of practicing mindfulness meditation

The question has come across in mind that when people are practicingmindfulness meditation, what is the most uneasiness for them? As the information in the chart (of Fg.11) has shown, there are differences in their view between male and female which we choose for making the example of the answer. From the point of view of male, they mostly consider time as the most uneasiness for them with 35 in its percentages, and then latter thing that could be their uneasiness is all above options, along with 32\%. Amongst them (male) environment are $33 \%$ uneasiness and technique are only $15 \%$ uneasiness ones for. Whereas for female practitioners have in their opinions that the most uneasiness that happened when they are taking meditation is environment conditions as the $36 \%$. They have thought that time, technique and all are $27 \%, 19 \%$ and $17 \%$ uneasiness things for them respectively.According to the information of the differences in their point of view between male and female, male seems to have time for practicing mindfulness less than female, it might be that male is staying in the social life such as having activities with friends more than female.

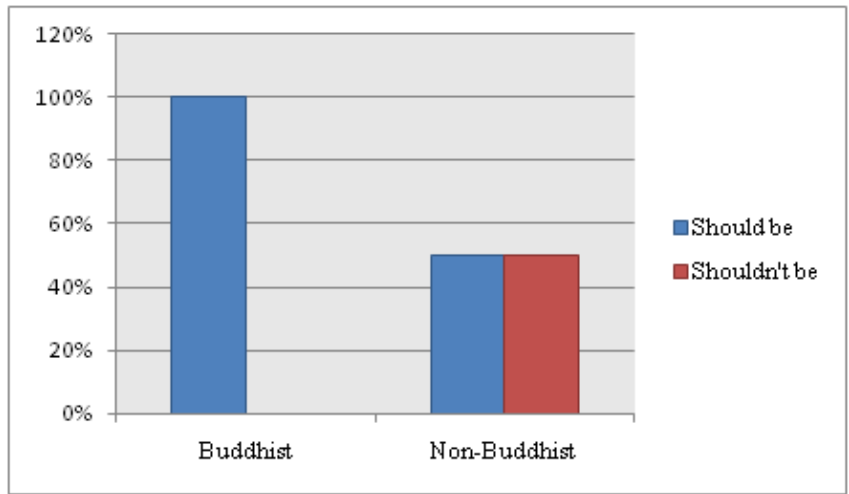

Fg. 12 - The purpose of practicing mindfulness meditation

The purpose of practicing mindfulness meditation comes to discover in this survey. The chart Fg.12, gives us the information as to why practicing meditation from the opinion of, of course, male and female. As to what is the purpose of them when taking meditation, for male, their purpose of taking meditation, most of them, is for peaceful mind with nearly $50 \%$ and for relaxation along with $35 \%$. Whereas for female, they give the point of view that their purpose of practicing meditation is peaceful and relax in equal with $46 \%$ of $100 \%$. Whereas, both of them, male and female, choose the others purpose for the last with $14 \%$ and $7 \%$ respectively.

As we have seen in the earlier information, the next question will comethat whether it could be compulsory for all human being? The answer might be arise from this survey is that, from Buddhist side, it should be made for the compulsory course, with $100 \%$, more than the one who did not agree which come in $0 \%$ in number of 160 respondents. Whereas the non-Buddhist side has thought that it should or should not are $50 \%$ and $50 \%$ the in percentages. This could indicate that if there is a compulsory course for taking meditation, they could also agree more than disagree.

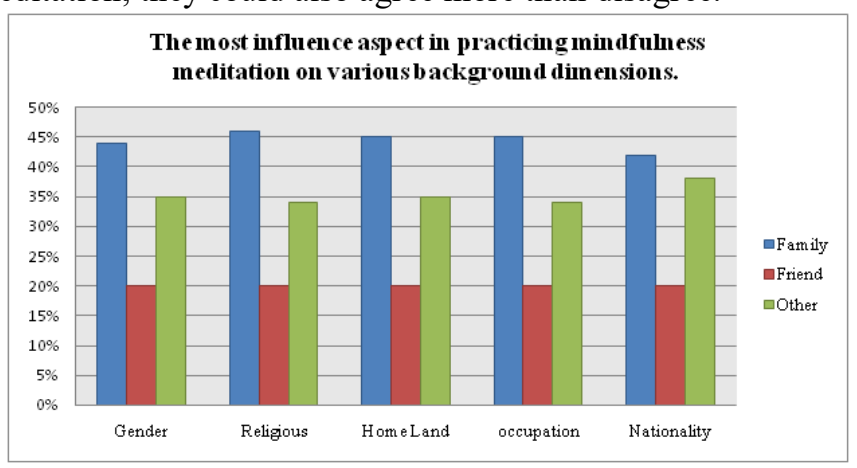

Fg.13- The most influence in practicing mindfulness

To the point as to who is the most important onestated in influence the practitioners in the first coming to join in mindfulness meditation. As in the chart above mentioned Fg.13, the result can be found from the point of view of several aspects their background, i.e. gender, religion, home land occupations where they are working, or even their nationality. Thefamily aspect is the most important one that is effect their way in joining mindfulness meditation with more than $41 \%$. Friends come to the next one that influencespractitioners for this question. The interesting thing in this survey about this question is that all of them, from any dimension, have the same percentages of 20 in which the other have influenced them in this way. From this survey, the result has consideration that family have most influence for practitioners because they may have been associated with their family for long times from since they were born. When one wants to promote the mindfulness meditation in its benefits they would focus on family first because of any country, any course, any place they were born, any religion and any gender they all have been related to their family mostly. That means in the promoting of meditation, the most effective way it could choose to start with, according to this survey, is that "family institution".

\section{EMPIRICAL BENEFITS OF MiNDFULNESS Meditation on Physical Diseases}

Implications of this survey project indicate comprehensive information how mindfulness impacts on mental and physical health problems. The results of this survey suggest that the increased mindfulness practice is associated along with physical health well-being. The outcomes of mindfulness practice are the most significant able to reduce physical diseases. Before mentioned the beneficial impact of this survey, it will be describe about some describing view of scholar that Grossman et al. (2004) explored the system of mindfulness based stress 
reduce (MBSR) and mentioned what they have found that 'mindfulness training might enhance general features of coping with distress and disability in everyday life, as well as under more extraordinary conditions of disorder or stress'. Grossman (2004) also found the other benefits of mindfulness practicing included the reduction of physical pain, impairments and diseases.

Practitioners who meditate mindfulness for long time can experience a significant reduction of mental suffering and physical symptoms. From this point of view of this survey, mindfulness practice can be seen as a preventive physical disease. Furthermore, the majority of this survey supports the notion that mindfulness practice for those along with physical disease lead to a reducing in levels of physical disease and symptom, and highest levels of well-being of mental and physical. Mindfulness practice can also assist in the prevention of physical health problems.

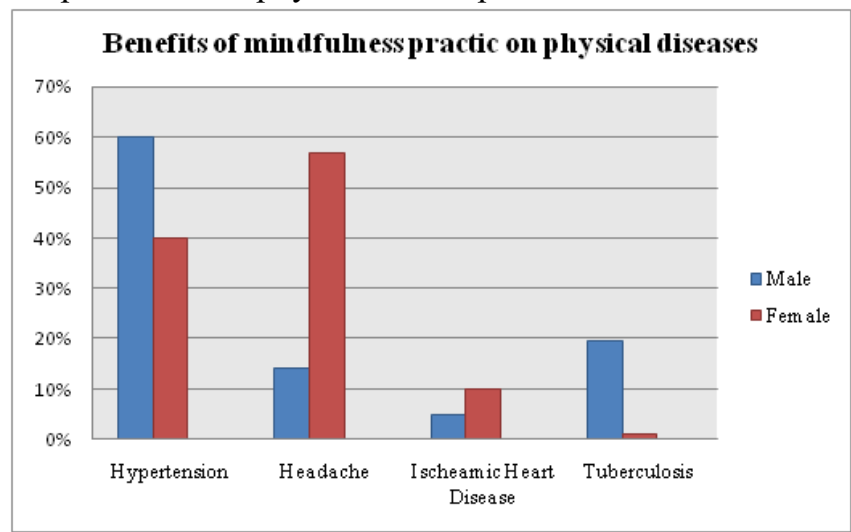

Fg.14- Benefits of mindfulness practice on physical diseases as gender aspect

The empirical benefits of this survey through questionnaires for respondents who are practicing for long time in the meditation center especially in India and Myanmar. Among them the results of this record for physical diseases have to mention right now. In the chart Fg.14, the most important beneficial results stated that $39 \%$ of 160 respondents can be reduced as hypertension (blood pressure) as practicing mindfulness meditation. $46 \%$ respondents have effects of mindfulness along with reduction of headache disease. $8.75 \%$ of 160 practitioners indicate reduction of ischeamic heart diseases and $5.5 \%$ have reduced the chronic disease of tuberculosis. As the demonstration of the gender, $60 \%$ of 41 male respondents can be reduced the disease of hypertension, $14 \%$ male have been responding able to reduce headache, $4.87 \%$ male are reducing the ischeamic heart disease and $19.5 \%$ can be reduced the chronic disease tuberculosis during and after practicing mindfulness meditation. For female $40 \%$ of 119 female respondents have been influenced the reduction of hypertension (high blood pressure), $57 \%$ female are recording due to reduce disease of headache, $10 \%$ are reducing the ischeamic heart disease, and only $1 \%$ of female can be reduced the chronic disease tuberculosis. Ifwe look upon the results of chat Fg.16, among physical diseases which are prepared only main four types in this survey questionnaires the types of diseases of hypertension (high blood pressure) and headache have been generally reduced by respondents as the most benefits of mindfulness practice on them.

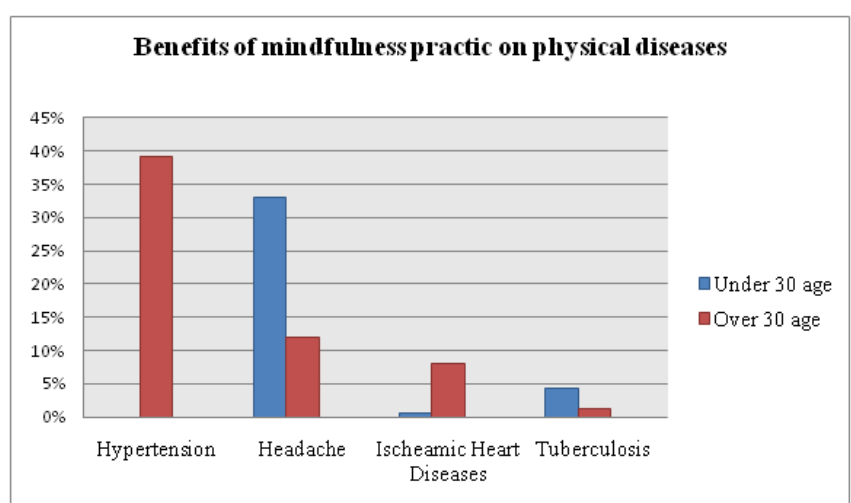

Fg.15-Benefits of mindfulness practice on physical diseases as age aspect

If the results have divided into two ages, there can be found in the chart Fg. 15, that the under 30 ages did not have any hypertension (high blood pressure) called $0 \%$ of 62 respondents. For the under 30 year old practitioners who had already faced the disease of headache could be reduced about $33 \%$ of 62 respondents. The next two results of this survey mention that $0.60 \%$ for ischeamic heart disease and $4.37 \%$ for the chronic disease of tuberculosis can be reduced by mindfulness practice the under 30 year old respondents. For over 30 age ones, $39 \%$ of 98 respondents have influenced of reduction of hypertension (high blood pressure). The $12 \%$ can be reduced the headache, the $8.12 \%$ are getting the benefits due to reduction of ischeamic heart diseases and the $1.25 \%$ responded the result of reducing the chronic disease of tuberculosis as they have the great benefits. As exploration of over view of this chart Fg.17, the over 30 age respondents are most significant on mindfulness practice along with reduction of physical disease more than younger ones. Especially, they have been severed the most benefits of mindfulness along with reduction of two physical diseases which are hypertension (high blood pressure) and headache.

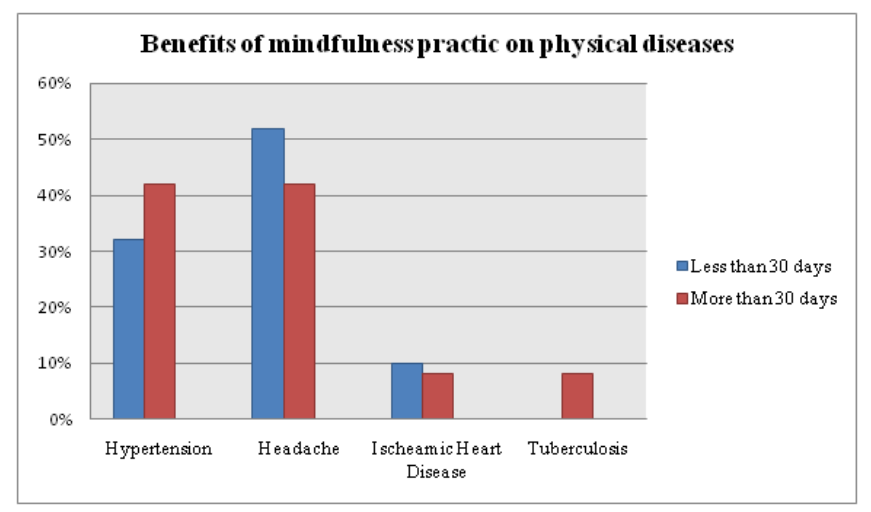

Fg.16- Benefits of mindfulness practice on physical diseases as period aspect

The final chart Fg.18, of this survey mentions about the benefits of mindfulness on respondents in accordance along with duration. In this Fg. 16, we can find the recoding results that 
$32 \%$ of 46 respondents are reducing the disease of hypertension (high blood pressure) for less than 30 days they have experienced.

The $52 \%$ respondents can reduce the headache disease during practice less than 30 days. The $10 \%$ and $0 \%$ respondents were indicated by results of survey to reduce ischeamic heart diseases and the chronic disease tuberculosis. Conversely, for more than 30 days of practitioners $42 \%$ of 114 respondents have the impact of mindfulness meditation able to reduce the hypertension (high blood pressure). For reduction of headache disease also $42 \%$ was recorded from practitioners of more than 30 days by practical survey. The percentages $(8 \%)$ of 114 respondents can be reduced ischeamic heart disease and tuberculosis chronic disease. Among them, if it divided into gender, 80\% of 15 male respondents can be reduced the hypertension (high blood pressure) during the time of less than 30 days, and $13.33 \%$ can reduce the headache disease, and $6.66 \%$ male respondents can reduce ischeamic heart diseases. For female practitioners of 114 respondents only $3 \%$ can be recorded the reduction of hypertension (high blood pressure) during less than 30 days practicing. The $21 \%$ female respondents are making decrease headache disease, $4 \%$ female are decreasing ischeamic heart diseases for less than 30 days meditation. Conversely, in the during more than 30 days practice, $29 \%$ of male respondents have recorded the results of decreasing of hypertension disease, $9 \%$ are indicating from the record that reduction of headache, $2 \%$ are decreased ischeamic heart diseases, and $17 \%$ male respondents can be reduced the chronic disease tuberculosis. For female practitioners there could be found in recording results that $31 \%$ respondents can decrease the hypertension (high blood pressure), 39\% female can be reduced the headache disease, $7 \%$ are indicated able to reduction of ischeamic heart diseases and only $0.87 \%$ of female respondents can be reduced the chronic disease tuberculosis. We are quite sure to warrantthat above mentioned account recording and results are empirical research survey used to try along with the important questionnaires by ourselves.

\section{CONCLUSION}

The purpose of the survey was to discover the relevant in mindfulness meditation in daily life of the respondents who were practicing mindfulness meditation as they can.The survey was conducted by means of a questionnaire given to practitioners of mindfulness to complete. The first part of the questionnaire dealt with the type of general information of gender, religion, home land, occupations and nationality. The second section was concerned with respondents' activities related to meditation which including many aspects as to taking meditation: how often the people practice meditation, what is purpose of the meditation, which places take the meditation, how influence in daily life, and meditation for free will or obligation as well as reduction of physical diseases.We can prove our hypothesis is that "meditation can be relevant to the people who interesting and practicing mindfulness meditation indaily life" as mentioned above.

If any conclusions may be drawn from the data, they are, perhaps, as fol $\neg$ lows. Through this survey we know that mindfulness meditation is good influence for the practitioners' lives. Especially, the results of this survey mentioned that the mindfulness meditation is more influence female practitioners than male. Furthermore, the aim of the mindfulness meditation is relaxation of mind called mental health and reduction of some diseases called physical health that practice of the meditation is influence in their lives.Moreover, under this circumstance, we have thought mindfulness meditation can be suitable solution of these stressful and painful situations.

\section{REFERENCES}

1. Catthasangiti, the Six Buddhist council. Ed.MahasstipatthānaSutta, DighaNikaya.(1954) All Tipitakas and Commentaries. Myanmar: Ministry of Religious affairs Press.

2. Gazzola. R. (2016) An Investigation of the Therapeutic Use of Mindfulness Practice, university of seattle, Canada.

3. Gethin, R. (2011). On some definitions of mindfulness.Contemporary Buddhism, 12(1), 263- 279.

4. Grossman, Paul and Van Dam, Nicholas T.(2011)Mindfulness, by any other name...: trials and tribulations of sati in western psychology and science, Contemporary Buddhism, http://dx.doi.org/10.1080/14639947.2011.564841(downloaded: 12 Aril 2019).

5. H.S.S. Nissanka (2018),Buddhist Psychotherapy, Buddhist Cultural Centre Publication., Dehiwala, Sri Lanka.

6. Kabat-Zinn. (2003) Mindfulness-based interventions in context: Past, present, and future. Clinical Psychology: Science and Practice.

7. Kabat-Zinn, J. (1994). Wherever you go, there you are: Mindfulness meditation in everyday life. New York: Hyperion.

8. Sidhu, Gurjeet, (2017). The Application of Western Models of Psychotherapy by Indian Psychotherapists in India: A Grounded Theory', Dissertations \& Theses. 377. http://aura.antioch.edu/etds/377 (downloaded 10.08.2019)

9. Siegel, D. J. (2007). The mindful brain: Reflection and attunement in the cultivation of well- being. New York: W.W. Norton.

10. VinaiUssivakul, (1996)An introduction to Buddhist Meditation for Results, Tippaywisut Ltd., Press, Bangkok.

11. Williams, K. (2015). Are we mindful of how we talk about mindfulness?The Psychologist journal, 28(3), 216-2017. 